in which it presses upon the neck of the gall bladder and the bile duct. However this may be, Newman showed a long while ago that if the abdomen is opened while the body is suspended in the erect position, the kidneys, which are already at their lowest normal point, fall much lower, even though these bands are uncut. Neither the fascia nor the fat is capable of maintaining the kidney in position when the influence of the abdominal pressure is removed.

The same may be said of the peritoneum. This is stronger on the right side than the left, owing to a special fold described by Jonnesco; but the kidneys glide behind it, and it is so easily displaced, even on the posterior wall of the abdomen, that they have no difficulty in pushing it before them. The surrounding organs check the movements of the kidneys and support them, but the kidneys are not in any way tied down or fastened by ligaments, like, for instance, the splenic flezure of the colon.

Kendal Franks described the kidneys as maintained in position by the combined pressure of the liver, acting from above and in front, and the colon acting from below and in front. But the liver and the colon, so far as intra-abdominal pressure is concerned, cannot be regarded as independent agents. Together with all the other organs enclosed by the peritoneum they form one soft elastic mass, pressing against the kidneys. If any one of the organs that form this mass changes in position or consistence, the rest-so long as the peritoneal cavity is unopened-compensate for it at once. There is no independent action until air has been admitted into the peritoneal cavity.

Glénard regards mobility of the kidneys as merely one of the phenomena of enteroptosis; but in the vast majority of cases in which the right sidney is movable, there is no displacement of the other viscera. When enteroptosis does occur, so that the liver rotates and the intestines sink, movable kidney may follow, owing to the disturbance of the intra-abdominal pressure. Even this, however, will only happen if there is at the same time defective development of the lumbar recesses.

The kidneys are maintained in position partly by the pressure in the extra-peritoneal space, partly by the way in which they fit into the lumbar recesses. Neither of these is sufficient without the other. Wolkow and Delitzin in particular, have drawn attention to the importance of the shape of the lumbar recesses. This is best shown by taking plaster casts of the posterior wall of the abdomen, while the body is suspended vertically. In the male they are deep, narrow, and pear-shaped, with the narrow end below. In the female, owing to the greater width of the pelvis, they are shallower and more cylindrical; and the difference in shape is almost always more marked upon the right side than upon the left. "This I believe to be due to a slight degree of torsion of the lumbar spine. In righthanded people there is usually a slight convexity towards the right in the dorsal spine. This entails an equivalent convexity towards the left in the lumbar region; and this, in its turn, necessitates a slight degree of rotation. The left transverse processes rotate backwards and deepen the lumbar recess upon that side. The right rotate forwards, and make the lumbar recess proportionately more shallow.

In a very large number of cases of movable kidney there is definite flattening of the right lumbar region. This, of course, cannot possibly be due to absence of the right kidney. Abdominal nephrectomy does not cause it. It really means that the vertebræ have rotated slightly, and is an indication of the cause of the displacement of the kidney, not a consequence of it.

The width of the lumbar recesses is one of the penalties which have followed the assumption of the erect attitude. If the history of the development of the renal organs in the race, as well as in the individual, is worked out, it is seen that, originally, they were distributed over the whole length of the head and body; and that by degrees, as their function became more specialised, they became concentrated together, until in their most perfect form they constitute a pair of organs, which, in the quadrupedal mammalia, are placed in a position of the greatest possible security. They lie close to the spine, immediately under the anticlinal vertebra, almost in the central axis around which all mevement of the trunk and limbs takes place. They, are buried in two deep and narrow recesses, protected by the lower ribs, and rest upon a horizontal sheet of fascia, which in its turn rests upon the peritoneal viscera. The slight degree of backward and forward movement which is necessitated by the action of the diaphragm takes place without the least risk, bat with the assumption of the erect attitude the greater portion of this security is lost. The backward and forward movement is altered to movement upwards and downwards, in the direction of the line of gravity. The peritoneal viscera and the supporting fascia lie in front and not below. The lumbar recesses, instead of being deep and narrow, become broad. The convexity forwards of the lumbar spine helps to make them still more shallow. The pelvis, especially in the female sex, becomes wider, so that the lower end of the recess becomes more open, and then the development of right-handedness causes the transverse processes of the lumbar vertebræ, which lie under the floor of the lumbar recess, to rotate. From a position of the greatest safety the kidneys are placed in one from which all security is gone; and it is not to be wondered at that comparatively trivial causes - an increase in the weight of the organ without a corresponding increase in the bulk, a sudden violent jerk, or a great lowering of the intra-abdominal pressure, can increase their normal range of mobility. The wonder is not that movable kidney occurs, but that it does not occur more often.

This accounts completely for the peculiar clinical distribution of movable kidney. It is certainly hereditary and common. It is present in women much more often than it is in men; and in women who have borne children more often than in those who have not. And it is much more common upon the right side than upon the left. None of the other reasons which have been assigned for this, such as the weight of the liver or the length of the renal vessels, deserve consideration.

[The lecturer, after detailing the different methods of troatment which have been advised, proceeded :]

The choice lies between wearing an abdominal belt and nephrorrhaphy. In my experience it is only in the milder cases than the former succeeds, and then it must be combined with massage and exercises calculated to strengthen the abdominal muscles. A belt braces the viscera together, and 80 steadies the displaced organ, but it cannot press the kidney back into its place or retain it there after it has been reduced if the patient stands upright and takes a deep breath. Pads are useless. For all cases in which there is manifest deformity of the lumbar region, associated with movable kidney, or in which there is real distress, even if the mobility attains no higher degree than the anteversion of Potain, I never hesitate to recommend nephorrhaphy. In the former case it is advisable, in order to save the kidney from hydronephrosis and other troubles. In the latter I believe it to be the most certain method of giving relief. I have never known it fail when properly carried out if the symptoms have not already lasted so long as to produce an indelible impression upon the patient's nervous system.

\section{A CONTRIBUTION TO THE SURGERY OF} PERFORATED GASTRIC ULCER,

WITH SPECIAL REFERENCE TO THE RESULTS OF OPERATION IN ULSTER.*

Bу A. B. MITCHELL, M.B., B.CH.,

Surgeon, Ulster Hospital for Women and Children ; Senior Assistantsurgeon, Royal Victoria Hospital, Belfast.

History and Statistics.

So far as I know, the first successful operation for perforating ulcer of the stomach was recorded by Kriege, a German surgeon, in 1892 . The first English success was reported by Mr. Morse, of Norwich, in March. 1894. The first Irish triumph falls to the credit of an Ulster surgeon, Dr. John Oampbell, whose operation was carried out in July, 1897 whilst the most brilliant result in the annals of this branch of surgery must be accorded to an Irishman, Dr. Myles, of Dublin, who operated with signal success on an old gentleman 


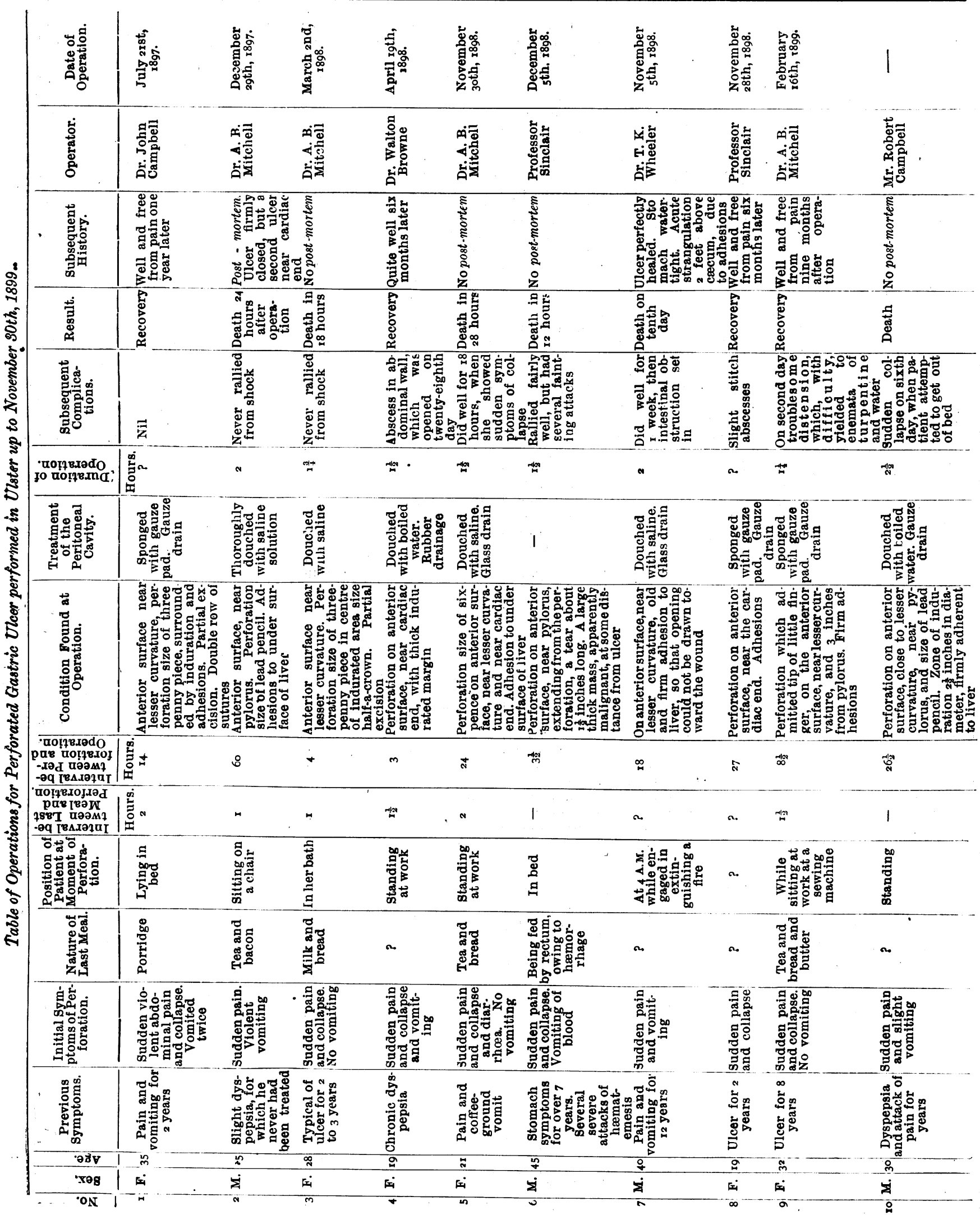




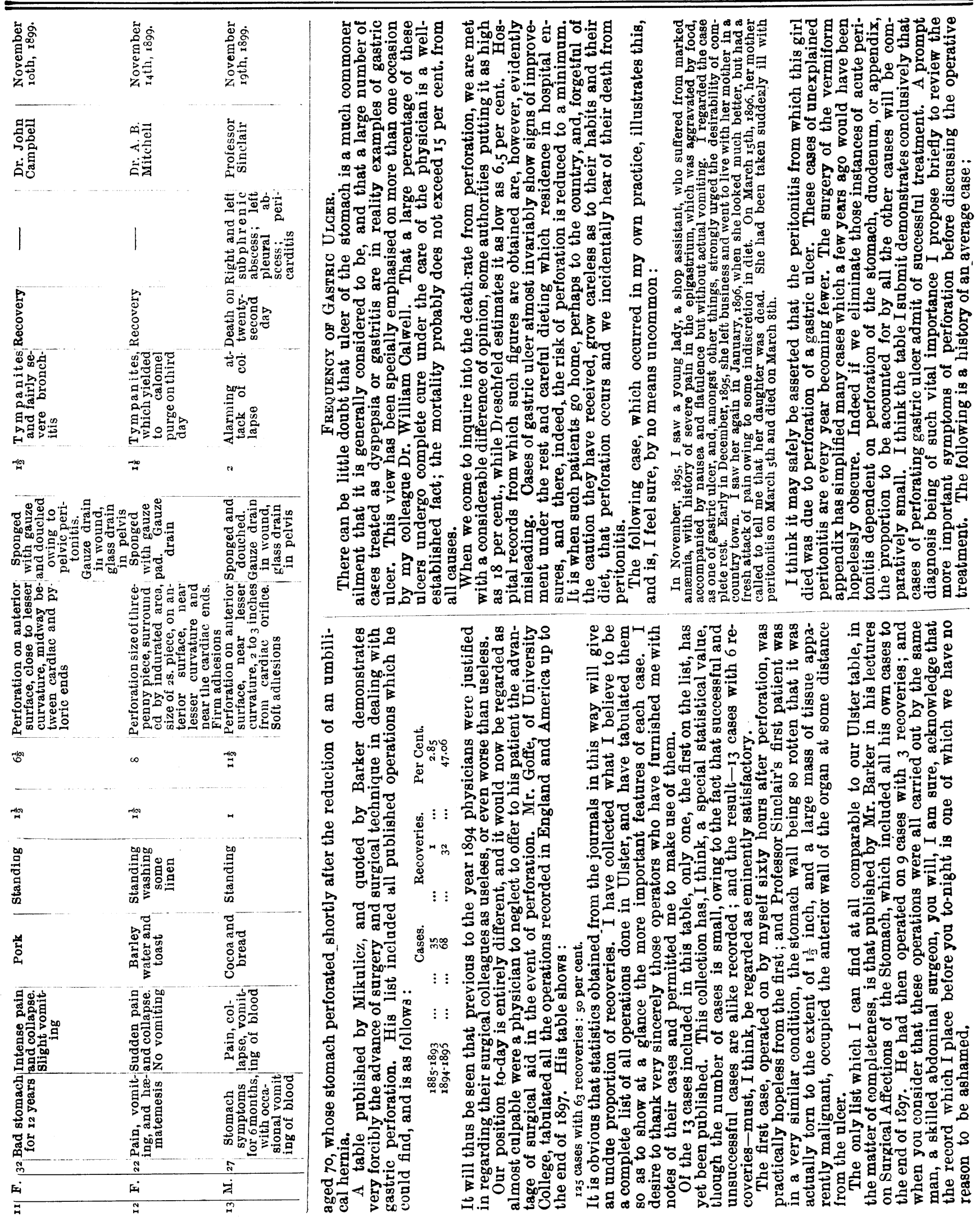


Symptoms of Perforation.

A patient, male or female, with or without a previous history of gastric symptoms, is suddenly seized with a violent abdominal pain, immediately followed by profound shock and collapse, and perhaps by vomiting. The pain, which may at first have been localised, rapidly becomes general. The abdomen becomes intensely tender, with its muscle hard and rigid. On percussion there is a tympanitic note all over; possibly there is a diminished area of hepatic dulness, especially in front. The bowels are inactive. The patient is cold and livid, with subnormal temperature, a quick, feeble pulse, thoracic respiration, anxious expression, and suffers from intense thirst. Soon a period of repose commences, when the sufferer feels distinctly easier, the pulse improves a little as the initial shock passes off, the abdominal retraction is replaced by slight distension, and liver dulness becomes markedly diminished. In a short time, however, the pain returns, tenderness increases, the temperature rises, and the patient exhibits all the signs of general septic peritonitis; or in the more fortunate cases the peritonitis is localised by adhesions and an abscess forms.

To the more important of these symptoms, pain, shock, vomiting, absence of liver dulness, and the period of repose $\dot{I}$ desire to refer at more length.

Pain comes on with startling rapidity, and generally commences in the epigastrium, but has been referred to the region of the gall bladder, simulating biliary colic, and even the right iliac fossa mimicking appendicitis. while in my last case it commenced in the region of the left clavicle, and gradually travelled down to the epigastrium. The tenderness with which it is associated affects the entire abdomen, but is almost invariably most marked over the epigastrium.

Shock is usually a very characteristic symptom, but is occasionally only slightly marked, and Charters Symonds records a case in which a patient on whom he operated and found a perforating ulcer had a pulse of 60 , and was free from pain. Shock, when present, appears to be due to

(x) Disturbance of the great sympathetic ganglia and peritoneum by the stomach contents, and I liave noticed the collapse was greater in those cases in which solid indigestible particles had

(2) The presence of gas in the peritoneal cavity, which, with the abdominal muscles powerfully contracted, ca.

Some observations kindly made for me by Dr. Calwell on my fourth case are of importance in this connection. He carefully observed the pulse from the conmencement of the anæsthesia to the completion of the operation. He found that the pulse, which reached 140 , and was very weak and irregular at the time of making the abdominal incision, promptly fell to 96 , and became fairly strong and regular when the peritoneum was opened and the gas allowed to escape.

In my fifth case there was very little free gas, and the opening of the peritoneum made no appreciable difference in the the pulse. In Professor Sinclair's third case I observed the pulse myself, and on the opening of the peritoneum and escape of gas it fell from 108 to 88 and distinctly improved in tone. Further observations on this point would be of considerable value, for if the escape of gas may be relied on to give some relief from shock, it obviously places in our hands a valuable weapon for obtaining temporary relief when the collapse is so profound as to exclude the practicability of a general anæsthetic or a complete operation. In such cases I would suggest a small incision under cocaine, opening of the peritonenm, removal of the gas, and insertion of a gauze drain, in the hope that a more thorough operation may be possible in a few hours. This procedure can be carried out in about five minutes, and cannot make matters worse. I followed this plan on a patient who was practically pulseless, rendering any complete operation impossible, whom I saw with my colleague, Dr. Wheeler, on April $3^{\text {rd, } 1899 . ~ T h e ~ h i s t o r y ~}$ pointed to perforating appendicitis, and $I$ cut down over this organ but failed to obtain any free escape of gas, and the little operation had no appreciable effect, though the patient herself thought she was slightly easier, she never rallied, however, from the original shock, and death occurred ten hours after the first symptom. Mr. Barker has, I think, since then recorded a case in which he followed the same line of treatment, unfortunately without any more favourable result.

Vomiting.-There seems to be a wide difference of opinion as to the frequency in which vomiting appears as an early symptom. Sir W. Broadbent says that "in the majority of cases there is vomiting and retching." Stawell estimates it as occurring in "about 70 per cent. of cases," while Dreschfeld, writing in Clifford Allbutt's System of Medicine, remarks that "vomiting is generally absent." You must, therefore, be be careful as to the importance you attach to this symptom. It was, as you will see, present in 8 out of the 13 cases in our list. In my first patient vomiting and retching were so severe however, that the case was looked upon as an example of acute intestinal obstruction, and treated by enemata for almost two days before be was sent into hospital. Vomiting, when present, has almost invariably this characteristic, that it is not long-continued, occurring probably only two or three times, thus presenting a striking contrast to the symptom when present in obstruction, where it is noted for its persistency.

Vomiting of blood as a symptom of perforation is very rare, but was present in the sixth and thirteenth cases in $\mathrm{my}$ table.

Abscnce of liver dulness has been called by Liebermeister a " pathognomonic sign of rupture of a hollow viscera." Unfortunately, like many other so-called pathognomonic signs, when weighed in the balance it is occasionally found wanting. Everyone who has carefully examined the abdomen of a number of patients suffering from chronic atonic dyspepsia or allied conditions, will recall instances in which liver dulness was greatly diminished, especially in front. On the other hand, it is quite possible to have a perforation in which the escape of gastric contents is so limited by adhesions that there may be no free gas in the peritoneal cavity. and in which the liver dulness will therefore remain unaffected. The sign however, is one that should alwass be looked for, and is of the greatest value when associated with other symptoms. Nevertheless, the following case shows that even then it cannot be absolutely relied upon:

A young woman was walking along the footpath on the Antrim Road one evening, about 8.30 P.M, when she was knocked down by a runaway horse, and the wheel of the vehicle went over her. She was promptly brought into hospital, and when I saw her about 9.3० P.M. I found her suffering from considerable shock, with severe abdominal pain, intense tenderness, rigid muscles, and absence of liver dulness. Her pulse was r20, and respiration shallow and thoracic. I immediately thought of rup the or ineel had apparently crossed the pelvis, thus diminishing the danger f viceral injury. we decided to await events. About ro P.M. several members of the staft happened to be at the hospita], and I took advantage of their presence to have a consultation. The result was that we came to the conclusion to recommend an exploratory incision. This, however, the patient resoJutely declined, and she left the hospital in about a week perfectly well.

Period of Repose.-Charters Symonds, who introduced this term, thus describes the condition :

In nearly all acute cases a stage is reached soon after the onset, marked by a cessation of all acute symptoms. The patient passes unaided by sedatives into a condition of repose. A lull takes place in the advance of the disease, or, more correctly, the relief from pain and sickness and the general sense of comfort experienced by the patient are interpreted as being signs of improvement. This period of repose, if wrongly interpreted, may lead to disaster, and if deepened by the regular administration of sedatives will almost certainly do so. It is in its early stage a eriod of safety, one in which operation is successful.

As illustrative of how deceptive this condition may prove, he relates the case of a girl of 20 suffering from a perforating gastric ulcer, who was at first refused admission to hospital then the physician who saw her deemed no operation necessary; yet a few hours later, when the abdomen was distended and operation was performed, it was too late.

$I$ have to confess that in my third case I was misled by this stage of repose; the girl, however, had received an opiate, and liver dulness was normal when I first saw her about ro o'clock at night. The following morning her condition was such as to admit of no hesitation, but the operation then undertaken was unsuccessful. Like most of our failures, however, it taught me a very sharp lesson, one which I am never likely to forget.

To sum up, the characteristic symptoms of perforation are sudden onset, violent pain, profound shock, intense tenderness, rigidly-contracted abdominal muscles, shallow thoracic respiration, and absence of liver dulness.

This certainly appears to be a sufficiently decisive list, and the great majority of cases will admit of an nccurate diagnosis; but occasionally nothing short of an exploratory in 
cision will enable us to arrive at an absolutely satisfactory opinion.

Early Operation.

That a few cases of true perforating ulcers recover without surgical aid is undoubted, and I believe I have seen one instance myself. All authorities, however, put the death-rate under these circumstances at over 95 per cent. In other words, without the interference of the surgeon, they are practically hopeless.

It will be worth while, therefore, to point out the chief factors which influence success. The first, and by far the most important, is early diagnosis and early operation. Once you have suspected that your patient suffers from the symptoms of perforation, there is not a moment to be lost. There is a slight difference of opinion amongst surgeons as to whether it is better to wait till the initial shock has passed off, but the trend of opinion seems to be against delay. Such, however. is the nature of these cases and the magnitude of the operation involved, that considerable time must of necessity be lost in obtaining surgical aid and in making the necessary preparations.

The importance of early operation is conclusively demonstrated by the following figures collected by Mr. Goffe :

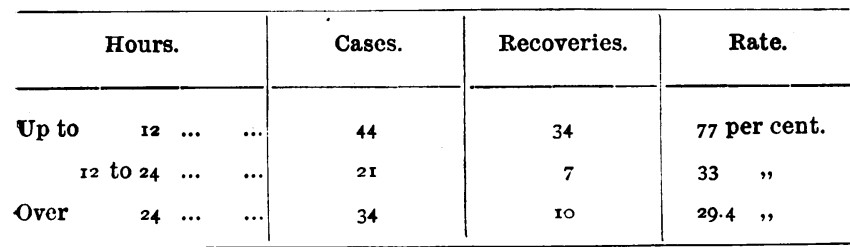

While these figures are all doubtless too high, being obtained from the various journals, they nevertheless furnish striking proof of the value of prompt operation, it being evident that if interference is delayed more than twelve hours the patient's chance of recovery is diminished more than balf. It cannot be too clearly understood, therefore, that the lives of these patients depend on the promptness and energy of the physician who first sees the case even more than on the skill of the operating surgeon. Above all things avoid the use of morphine if possible till a final diagnosis is made and the line of treatment decided. The other points of importance to be noted previous to operation are: (1) The nature of the last meal taken; (2) the interval of time between the last meal and the occurrence of perforation; (3) the position of the patient at the time of perforation.

It is a well-known fact that the stomach contracts firmly and vigorously on indigestible food in an endeavour to force it through the pylorus, which in its turn contracts tightly to prevent the escape of the partially-digested material. Should a perforation occur under these circumstances the food is liable to be driven a considerable distance into the peritoneal cavity, and the contents of the stomach are more likely to reach the pelvic regions if perforation occurs whilst the patient is in the erect position.

In point of time perforation usually occurs about two hours after a meal. The longer this interval the more complete will be the process of digestion and the smaller the quantity of food remaining in the stomach. The danger dueto extravaeation will of course be proportionately diminished.

The Operation.

As I have had the good fortune to have been present at no fewer than eleven out of the thirteen cases, I think I may with advantage refer to some of the more important lessons which have impressed themselves on my mind.

1. The incision in the first instance should be a fairly small one, and the peritoneal opening as small as possible in order that the gas may escape slowly. Once all doubt as to the diagnosis has been set at rest, the wound may be rapidly enlarged to the required length by scissors.

2. The best guide to the ulcer seems to be the presence of adhesions. Haviug drawn gently on the stomach, you find that in some direction it is more fixed than in others. On running the finger down to this area you will generally come on a zone of induration in the midst of which the ulcer is almost certain to be found when the adhesions have been separated. Of course, cases occur in which perforation take place so rapidly alter the onset of the ulcer that there is no time for aahesions, or for induration, but they are very rare.

The bubbling up of fluid and other gastric contents is not necessarily a guide to the seat of perforation. In Professor Sinclair's last case the least pressure caused free bubbling near the pploric end of the stomach of contents which had escaped from an ulcer near the cardiac end, and which was ultimately found concealed by adhesions to the under-surface of the left lobe of the liver.

Should a careful search fail to find the ulcer on the anterior surface, it will be necessary to open the lesser sac of peritoneum, and this can be most easily done by tearing through the layers of the great omentum, when the posterior surface can be examined. In at least two of the cases in my table it was necessary to divide the left rectus transversely in order thoroughly to expose the ulcer.

Having found the ulcer the next thing is to bring it as well out through the wound as possible; and though I have read of several cases in which this has been done with ease, my own experience has not been so fortunate. With but one exception it was not possible even to draw the ulcer up to the wound; while in one of my own cases, and in that operated on by Dr. Wheeler, the sutures had to be inserted under circumstances of the greatest difficulty. The stomach in both these instalices was very firmly adherent in the region of the transverse fissure of the liver, and the operator had to work with his needle at the bottom of a deep wound.

Treatment of the Ulcer. - Surgeons do not as a rule make any attempt to excise the ulcer, as this involves considerable risi of hæmorrhage, bringing in its train delay and increased shock. Occasionally a partial excision is carried out. This was done by Dr. Campbell in his first case, and in my second patient the tissue round the perforation was so friable that it would not hold a suture, and I was obliged to remove a portion of it. Generally a continuous suture perforating all the coats of the stomach is employed to close the opening, and this in its turn is buried by a row of Lembert sutures.

Cleansing of the Peritoneal Cavify.-This may be attempted in two ways:-1. By sponging with sterilised gauze pads, which are wonderfully effectual in removing coarse material, and rapidly soak up fluid. 2. By thorough douching with boiled water or normal saline solution. Most surgeons, I think, now favour the former method, though douching stil has powerful advocates. Dr. Myles speaks strongly in its favour and says that it acted as a powerful stimulant to his old patient (70 years), who was profoundly collapsed, but at once rallied under the influence of the warm saline douche. In the 6 successful cases in my table you will see that gpong ing alone was employed in 4, douching alone in 1 , whilst in in which the gastric contents had reached the pelvis both methods were employed. Of my own cases, 3 in which I employed the douche were unsuccessful, but they were at the game time the most unfayourable of the five. Neither method will thoroughly cleanse the abdominal cavity, and were it not the peritoneum has wonderful powers of resistance and absorption we could never hope to approach there cases with any prospect of success.

Drainage. - I do not think any surgeon would now neglect to employ a drain after this operation. A gauze drain is gradually taking the place of the oldermethod, and a strand of this. material should be introduced so as to lead down to the line of suture, and one into each subphrenic space, as it is here that abscess most frequently occurs. The only point in reference to these drains is the rapidity with which they form ad. hesions; and for the benefit of any surgeons who may not yet have employed them, I would point out that while they are often very difficult to remove on the third or fourth day, they will, if left alone, gradually loosen themselves, and slip out without difficulty at the end of a week.

\section{AFTER-TREATMENT.}

The operation being completed, the dangers which still face the patient are both numerous and grave. A short reference to the most important must suffice.

Shock.-First of all comes shock, which is the commonest cause of death, and must be energetically dealt with ; external warmth, elevation of the lower limbs, strychnine hypodermically, and stimulants by the rectum will all be called 
for. From the outset, shock is very severe, and when we add to this the effect of a difficult and dangerous operation, it is obvious we are taxing our patient's resistance to the utmost. It is therefore of supreme importance that every effort should be made to complete the operation as rapidly as possible. In our modern zeal for thorough asepsis, I am afraid we are rather inclined to under-estimate the value of rapid surgical work. Here, however, the question of time has an intimate bearing on the prospect of success, and we cannot afford to neglect it.

Peritonitis.-Our next complication is peritonitis. It is always present to some extent. Its severity will depend on the nature of the extravasated material and the throughness with which cleansing has been carried out.

Tympanites is another very troublesome complication. It occurred in two of my cases and in one of Dr. John Campbell's. It must be promptly dealt with, and here we are face to face with a serious difficulty. A purge unfortunately is precluded by the state of the stomach. It must, therefore, be combated by turpentine enemata, use of the rectal tube, and gentle movement of the patient from one side to the other. Should these measures fail, a purge must be risked, and I gave my last case 5 grains of calomel on the third day, and in this way obtained the necessary relief.

Abscess.-Subphrenic abscess is another very fatal complication, and is commoner on the left side, extending to the splenic area. Deaths from this cause are reported by Whipple, Jones, Barker, Roughton, and others.

Empyema.-Mr. Morse records a case in which a left pleural abscess developed. This was opened and drained, and the patient recovered.

Hemorrhage.-Mr. Barker mentions one of his cases, which made an apparently good recovery from the operation, but was suddenly seized on the tenth day with a violent attack of hæmatemesis, from which he never rallied.

Other Causes of Death.-Among the causes of death which have been recorded may be mentioned pneumonia, hepatic abscess, and rupture of a second ulcer. Intestinal obstruction was the cause of death in the case operated on by $\mathrm{my}$ colleague, Dr. Wheeler. The patient died on the tenth day and the post-mortem notes made by Dr. Lorrain Smith are as follows :

Marked abdominal distension, the abdominal wound being torn open by the distended small intestine. Stomach was firmly adherent to the diaphragm, liver, spleen, and some loops of small intestine. On tracing the small intestine downwards, congestion and distension were well marked till a point was reached $2 \mathrm{ft}$. above the ileo-cæcal valve. Here the bowel was bent sharply back on itself, forming a $\mathbf{V}$-shaped bend, with the limbs of the $\mathbf{V}$ firmly bound together, and the apex adherent to the anterior abdominal wall, causing a complete obstruction of the gut at this point. The $2 \mathrm{it}$. of small intestine below this, and all the large intestine was collapsed and empty. When the stomach was removed the opening was found firmly closed; the sutures were in position, and on distending the viscus with water there was no leakage whatever. On opening the stomach and examining the inner surface no trace of the ulcer co zld be found. The mucous membrane was perfectly healed, leaving no sign what

This an exceedingly interesting record, demonstrating very clearly two important points :

I. How rapid and thorough is the effort at repair.

2. The great extent of the adhesions which we may expect to follow this operation.

The first point is of importance as forming a guide to the time after operation at which we may allow food freely by the mouth. In my last case the patient had sips of water after forty-eight hours, peptonised milk on the third day, tea on the fifth, and fish on the seventh day, and chop at the end of a fortnight.

The second point bears very directly on the question of the influence of adhesions on the functions of the stomach.

Adhesions.- It has been recently pointed out that adhesions are one of the causes of gastric pain. It is, however, a curious fact that patients who have been successfully operated upon are, in the majority of instances, practically free from pain and gastric trouble. This is probably explained by the fact that while slight adhesions are liable to stretch, those following operation are so extensive and firm that stretching is almost impossible.

Since the above paper was written the following case has come under my care :

Miss L. D., aged 2o, had suffered for ab out four years from gastric disorder, her prominent symptoms being (1) Severe pains in the epigastrium coming on about half an hour after food, relieved by lying on the left side and on the back; (2) nausea: (3) vomiting only about once a week, and this always gave relief to the pain; she never vomited any blood; (4) anæmia and constipation; her diet for more

imited to milk foods, and even these caused pain. Perforation.- On January. 23rd, I900, during a visit to a friend's houter and a cup of tea, while sitting in a chair, she was suddenly seized with a violent pain in the epigastrium, and felt very sick and faint, but did not vomit. She was helped home-a distance of about 200 yards-" "half walking, half carried." Dr. Ledlie. who saw her almost immediately, found her in a very collapsed condition, with a rapid thready pulse. He suspected perforation, and Professor Whitla, who saw her in consultation, confirmed his opinion, and she was promptly sent to hospital, her home surroundings being unsuitable for operation

Condition on Admission. - About rr.45 P.M. her condition was as follows : Colour fair; initial shock had passed off, and she said she felt much better; pulse rr8, but small ; pain over the abdomen was very slight, though there was distinct tenderness in the epigastrium just below the xiphoid ; abdomen was slightly distended. Liver dulness was normal, it being impossible to differentiate stomach or colic resonance. Respirait being impossible to differentic

Operation.-This was undertaken five hours after perforation. An ncision was made from the xiphoid to the umbilicus. On opening the peritoneum there was an immediate escape of stomach contents with vidence of localised peritonitis. The stomach was adherent to the under-surface of the left lobe of the liver. On gently separating the adhesions, a perforation the size of a threepenny-piece was found, surrounded by a zone of induration larger than a crown-piece : this area was pale and friable, and the tissue of which it was formed failed to hold a suture. The ulcer was situated on the middle of the anterior surface of was closed by a single row of Lembert's sutures, end. The perforation was closed by a single row of Lembert's sutures, 15 in number, and about 42 inches long, the entire indurated area being enfolded. There was no theroughly sponged with gauze pads, three gauze drains were inserted. one into each subphrenic space and a third leading down to the line of suture, and the wound closed with silkworm gut. The operation lasted an hour and a-half.

Afler-history.-The patient suffered from troublesome vomiting for two days, which yielded to a hypoderinic injection of morphine bimeconate gr. $\frac{1}{x}$. Bhe passed urine the day following operation (this I always regard as a very favourable sign), and her recovery was uneventful. The gauze drains were removed on the sixth day. She was fed every four hours by peptonised nutrient enemata, consisting of bovril 3iv, whisky $38 s$, alternating with peptonised milk ऊiv, I egg, whisky 3 ss. Nutrient supposi-
tories were also employed, and water was given by the mouth on the
second day, peptonised milk in small quantities on the fourth day ; on the second day, peptonised milk in small quantities on the fourth day ; on the fifth day some Brand's essence and some cocoa; on the sixth day Benger's food. After ten days rectal feeding was discon

able to take tea, fish, and thin bread and butter. Three weeks after the operation she was able to be up, and could eat
chicken, fish, and chop without the least discomfort for the first time for himen, ish, and ch

In this case the ulcer was on the anterior surface, and the pain was relieved by lying on the back. In my third patient the pain was greatly aggravated by lying on the back, and I rather expected to find a posterior ulcer, but the lesion was on the anterior surface. The relief of pain by any special position can therefore not be relied on to give trustworthy information as to the seat of the ulcer.

This brings the total number of cases operated on in Ulster up to 14 , with 7 recoveries. My own operations amounted to 6 , with 3 recoveries.

REFERENCES.

Barker, Surgical Affections of the Stomach, $1898 .^{*}$ Barling, Ingleby Lecture, IV. Clifford Allbutt, System of Medicine, vol. jii, p. 535. Broadbent, BRITISH MEDICAL JodRNAL, November 3oth, 1897. Lundie, Edinburgh Hospital Record, vol. iv, p. 485. Stawell, Bartholomew Hospital Gazette, March, 1898. Myles, Transactions of the Royal Acadewy of Medicine in Ireland. Campbell, BRITISH MEDICA L JodRNAL, July $x 6$ th 1898, p. 150. Morse, Ibid., February 13 th, 1897. Winston Hall, Ibid., January 9th, 1892. ton, Ibid., July gth, 1898. Symonds, Ibid., March 4 th, 1899.

\section{PERFORATING GASTRIC ULCER, WITH NOTES OF TWO SUCCESSFUL CASES AFTER OPERATION.}

By P. RHYS GRIFFITHS, M.B., B.8.LoND., President of the Cardiff Medical Society and Surgeon to the Cardiff Infirmary.

THERE is no department in which surgery has made such remarkable advances within recent years as in the treatment of abdominal disease, and it may safely be predicted that with increasing accuracy in diagnosis the brilliant results which have hitherto been achieved will be wholly eclipsed. Fifteen zears ago a case of perforating ulcer of the stomach was regarded as almost of necessity fatal, and the physician was powerless to check the course of the disease, and only 